\title{
STK10 missense mutations associated with anti-apoptotic function
}

\author{
KAZUTAKA FUKUMURA $^{1,2}$, YOSHIHIRO YAMASHITA ${ }^{4}$, MASAHITO KAWAZU $^{1}$, EIRIN SAI ${ }^{1}$, \\ SHIN-ICHIRO FUJIWARA ${ }^{5}$, NAOYA NAKAMURA ${ }^{6}$, KENGO TAKEUCHI $^{7}$, MIZUO ANDO ${ }^{1}$, \\ KOHEI MIYAZONO ${ }^{2}$, TOSHIHIDE UENO ${ }^{4}$, KEIYA OZAWA ${ }^{5}$ and HIROYUKI MANO ${ }^{1,3,4,8}$
}

\begin{abstract}
Departments of ${ }^{1}$ Medical Genomics, ${ }^{2}$ Molecular Pathology and ${ }^{3}$ Cellular Signaling, Graduate School of Medicine, The University of Tokyo, Tokyo 113-0033; ${ }^{4}$ Division of Functional Genomics and ${ }^{5}$ Department of Hematology, Jichi Medical University, Tochigi 329-0498; ${ }^{6}$ Department of Pathology, Tokai University School of Medicine, Kanagawa 259-1193; ${ }^{7}$ Pathology Project for Molecular Targets, The Cancer Institute of the Japanese Foundation for Cancer Research, Tokyo 135-8550; ${ }^{8}$ CREST, Japan Science and Technology Agency, Saitama 332-0012, Japan
\end{abstract}

Received May 8, 2013; Accepted June 12, 2013

DOI: 10.3892/or.2013.2605

\begin{abstract}
Peripheral T-cell lymphoma (PTCL) is an aggressive lymphoma with a 5 -year overall survival rate of $<30 \%$. To identify carcinogenesis-related genes in PTCL, we conducted high-throughput resequencing of target-captured cDNA in a PTCL specimen, revealing a total of 19 missense mutations among 18 independent genes. One of such substitutions, c. $2201 \mathrm{G}>\mathrm{A}$ in STK10 cDNA, replaces an arginine residue to a histidine $(\mathrm{R} 634 \mathrm{H})$ in the encoded protein. Of note, while wild-type STK10 suppresses NF- $\kappa$ B activity and potentiates dexamethasone-induced apoptosis, the R634H change significantly decreases such pro-apoptotic activity. This c.2201G $>$ A change of STK10 was also identified in another PTCL specimen, but now registered as a single nucleotide polymorphism in the latest dbSNP database. Furthermore, other somatic mutations of STK10 have been reported, and we now reveal that some of them (L85P and K277E) have more profound anti-apoptotic effects compared to R634H. These results suggest that STK10 functions as a tumor suppressor gene, and that dysfunction of STK10 activity either through polymorphism or somatic mutations may confer anti-apoptotic effects contributing to carcinogenesis.
\end{abstract}

\section{Introduction}

Various genetic alterations such as point mutations, insertions/ deletions (indels) and gene fusions directly participate in human carcinogenesis. Some of such changes confer growth advantage to cancer cells, and targeting their encoded proteins is one of the most effective means to treat cancer. Activating mutations in EGFR (1) or the gene fusion between EML4 and

Correspondence to: Professor Hiroyuki Mano, Department of Cellular Signaling, Graduate School of Medicine, University of Tokyo, 7-3-1 Hongo, Bunkyo-ku, Tokyo 113-0033, Japan

E-mail: hmano@m.u-tokyo.ac.jp

Key words: tumor suppressor gene, apoptosis, NF-кB
$A L K$ (2) are, for instance, identified in subsets of non-small cell lung cancer (NSCLC), and reagents targeting EGFR or ALK are proved clinically effective against NSCLC positive for the corresponding genetic lesions $(3,4)$.

Peripheral T-cell lymphoma (PTCL) is a subdivision of non-Hodgkin's malignant lymphoma (5). In contrast to the improved prognosis for individuals with B-cell lymphoma with recent treatment modalities, most subtypes of PTCL still have a 5 -year survival rate of only $<30 \%$ (6). Molecular mechanism for PTCL carcinogenesis is mainly enigmatic, except for the facts that i) a part of PTCL is associated with infection with Epstein-Barr virus (EBV) or human T-lymphotropic virus type I (HTLV-I), and ii) activating ALK fusions are found in the anaplastic large cell lymphoma (ALCL) subtype. Given the fact that an ALK inhibitor is effective in the treatment of ALCL (7), identification of essential growth drivers in other subsets of PTCL is urgently needed.

While exome sequencing of cancer specimens is used for the detection of somatic mutations in the cancer genome, such approach fails to detect gene fusions, because gene fusions usually take place at intronic regions. To simultaneously detect point mutations/indels/gene fusions in a single experiment, we previously reported the 'cDNA capture system' that conducts massive resequencing on purified cDNAs for cancer-related genes (8). Herein we applied such technology to the cDNAs isolated from a PTCL specimen, and discovered a STK10 amino acid substitution that turned out to exert anti-apoptotic effects. While this amino acid change was recently deposited as a single nucleotide polymorphism (SNP) in the 1000 genome database (http://www.1000genomes.org), we also confirmed that other nonsynonymous, somatic mutations within STK10 confer marked anti-apoptotic activity. These results suggest that STK10 may contribute to carcinogenesis, either through polymorphism or somatic mutations, by suppressing apoptotic signaling in cancer.

\section{Materials and methods}

Cell lines and specimens. PTCL specimens were collected in Fukushima Medical University and The Cancer Institute. 
A human embryonic kidney 293T (HEK293) cell line was obtained from American Type Culture Collection (ATCC: Manassas, VA, USA), and maintained in Dulbecco's modified Eagle's medium-F12 medium (Invitrogen, Carlsbad, CA, USA) supplemented with $10 \%$ fetal bovine serum (FBS) and 2 mM L-glutamine (both from Invitrogen). An IL-2-dependent mouse cytotoxic T-cell line, CTLL-2, and a human T-cell line, Jurkat, were both obtained from ATCC, and maintained in RPMI1640 medium (Invitrogen) supplemented with 10\% FBS. Mouse recombinant IL-2 (Peprotech, Rocky Hill, NJ, USA) was added to the culture medium of CTLL-2 at the concentration of $2 \mathrm{ng} / \mathrm{ml}$. Total RNA was extracted from cell lines and a PTCL specimen with an RNeasy mini kit (Qiagen, Valencia, CA, USA), and was subjected to reverse transcriptase (RT) with an oligo-dT primer. Written informed consent was obtained from the subjects who provided cancer specimens, and the study was approved by the human ethics committee of the University of Tokyo, Jichi Medical University, Fukushima Medical University, and The Cancer Institute of the Japanese Foundation for Cancer Research.

Resequencing of target-captured cDNA in a PTCL specimen. Resequencing with a custom cDNA-capture system was performed as previously described (8). In brief, RNA probes of 120 bases were designed to interrogate cDNAs of 906 human protein-coding genes, and were synthesized by Agilent Technologies (Santa Clara, CA, USA). Hybridization of cDNA fragments isolated from a PTCL specimen to the RNA probes was performed according to the protocols recommended for the SureSelect Target Enrichment system (Agilent Technologies). Purified cDNA fragments were then subjected to deep sequencing with a Genome Analyzer IIx (GAIIx; Illumina, San Diego, CA, USA) for 76 bases from both ends by the paired-end sequencing system.

Plasmid construction. The full-length cDNA for the wild-type or the R634H mutant of STK10 was amplified by PCR from cDNA of a PTCL specimen. The cDNAs for other mutant forms of STK10 were further generated by a polymerase chain reaction (PCR)-based mutagenesis approach. The cDNA for PLK1, IKK- $\alpha$, IKK- $\beta$ or IKK- $\gamma$ was amplified by PCR from our cancer cell lines.

Luciferase-based reporter assay. HEK293 cells were transfected with the expression plasmids, a promoter-less Renilla luciferase plasmid pGL-4.70 (Promega, Madison, WI, USA), and firefly luciferase-based reporter plasmid for Fos (pFL700) (9), Myc (pHXL) (10), NF-кB (Stratagene, La Jolla, CA, USA), Bcl-xL (11), Notch (pGa981-6) (12), Wnt (TOP-flash, Upstate Biotechnology, Lake Placid, NY, USA) or Rho (pSRE.L) (13) signaling pathway. Luciferase activities were determined with the Dual-Luciferase Reporter Assay System (Promega), and the firefly luciferase activities were normalized to the Renilla luciferase activities.

Quantitative real-time RT-PCR. Quantitative real-time RT-PCR was performed with QuantiTect SYBR Green PCR kit (Qiagen) and an Applied Biosystems 7900HT Fast RealTime PCR System. The PCR conditions include the first incubation at $50^{\circ} \mathrm{C}$ for $2 \mathrm{~min}$ and then $95^{\circ} \mathrm{C}$ for $15 \mathrm{~min}$, followed by 60 cycles of $94^{\circ} \mathrm{C}$ for $15 \mathrm{sec}$ and $60^{\circ} \mathrm{C}$ for $30 \mathrm{sec}$ and $72^{\circ} \mathrm{C}$ for $1 \mathrm{~min}$. Relative expression levels of target mRNAs to $G A P D H$ were normalized to those in the mock-transfected cells. The primer sequences used for the PCR reactions were: 5'-TTCATTCCTGGCAAGTGGATCATT-3' and 5'-ATGG CAGCATCATTGTTCTCATCA-3' for TLR2, 5'-TGACAAC CTTCTGGTTGGTAGGGA-3' and 5'-CCAAGGTCATGGT TGTCCAAAGAC-3' for BCL2, 5'-AAGAATCACCAGCA GCAAGTGTCC-3' and 5'-TTGGGTTGTGGAGTGAGTG TTCAA-3' for CCL2, and 5'-CCAGGTGGTCTCCTCTGAC TTCAA-3' and 5'-CACCCTGTTGCTGTAGCCAAATTC-3' for $G A P D H$.

Antibodies, immunoprecipitation and immunoblotting. Antibodies used in this study were: anti-FLAG M2 (SigmaAldrich, St. Louis, MO, USA), anti-PLK1 (Santa Cruz Biotechnology, Santa Cruz, CA, USA), anti-phospho-PLK1 (BD Pharmingen, San Jose, CA, USA), anti-ACTB (Cell Signaling Technology, Danvers, MA, USA), anti-mouse IgG and anti-rabbit IgG (both from GE Healthcare, Piscataway, NJ, USA). HEK 293 cells were transfected with the appropriate expression vectors, and then lysed in lysis buffer [1\% NP-40, $50 \mathrm{mM}$ Tris- $\mathrm{HCl}$ (pH 7.4), $150 \mathrm{mM} \mathrm{NaCl}, 1 \mathrm{mM} \mathrm{NaF}, 1 \mathrm{mM}$ $\mathrm{Na}_{3} \mathrm{VO}_{4}, 1 \mathrm{mM}$ phenylmethylsulfonyl fluoride and aprotinin]. Target proteins were purified by incubation of the cell lysates with appropriate antibodies and Protein G Sepharose Fast Flow (Sigma-Aldrich) for $3 \mathrm{~h}$ at $4^{\circ} \mathrm{C}$. Immunoblot analyses were visualized with SuperSignal West Femto Maximum Sensitivity Substrate (Thermo Scientific, Waltham, MA, USA).

In vitro kinase assay. Immunoprecipitates were washed with kinase buffer [50 mM NaCl, $50 \mathrm{mM}$ Tris- $\mathrm{HCl}$ ( $\mathrm{pH} 7.4$ ), $10 \mathrm{mM} \mathrm{MgCl}_{2}, 10 \mathrm{mM} \mathrm{MnCl}_{2}$ and $\left.0.1 \mathrm{mM} \mathrm{Na}_{3} \mathrm{VO}_{4}\right]$, and then incubated in kinase buffer containing $\left[\gamma-{ }^{32} \mathrm{P}\right] \mathrm{ATP}$ (PerkinElmer, Boston, MA, USA) and histone H2A protein (New England BioLabs, Ipswich, MA, USA) for $30 \mathrm{~min}$ at room temperature.

Dexamethasone-induced apoptosis assay. CTLL-2 cells were infected with retrovirus expressing STK10 and the blasticidinresistant gene, and cultured under the presence of $10 \mu \mathrm{g} / \mathrm{ml}$ blasticidin (InvivoGen, San Diego, CA, USA). Blasticidinresistant cells were then treated with $1 \mu \mathrm{M}$ dexamethasone (Sigma-Aldrich), and cell number was determined every $24 \mathrm{~h}$ with CellTiter-Glo Luminescent Cell Viability Assay (Promega). At $72 \mathrm{~h}$ after treatment with dexamethasone, CTLL-2 cells were collected and subjected to apoptosis assay with flow cytometry (FACSCanto II; BD Biosciences, San Jose, CA, USA) using Annexin V/propidium iodide (PI) staining kit (eBioscience, San Diego, CA, USA).

\section{Results}

Identification of STK10(R634H) in a PTCL specimen. To identify transforming genes in PTCL, cDNAs for cancerrelated genes $(n=906)(8)$ were isolated from a PTCL specimen obtained from a 25 year-old male negative for EBV and HTLV-I infection, and subjected to deep sequencing with GAIIx. We thus obtained a total of 69,552,278 independent, high-quality reads that mapped to 868 cDNAs $(95.8 \%)$. 
Table I. Nonsynonymous mutations detected in a PTCL specimen.

\begin{tabular}{|c|c|c|c|c|c|}
\hline $\begin{array}{l}\text { Gene } \\
\text { symbol }\end{array}$ & $\begin{array}{c}\text { GenBank } \\
\text { accession no. }\end{array}$ & $\begin{array}{c}\text { Nucleotide } \\
\text { change }\end{array}$ & $\begin{array}{c}\text { Read } \\
\text { coverage }\end{array}$ & $\begin{array}{l}\text { Mismatch } \\
\text { reads }(\%)\end{array}$ & $\begin{array}{c}\text { Amino acid } \\
\text { change }\end{array}$ \\
\hline$A X L^{\mathrm{a}}$ & NM_001699 & c. $1506 \mathrm{G}>\mathrm{T}$ & X156 & 59.0 & W439L \\
\hline$C D C 7$ & NM_003503 & c. $1732 \mathrm{C}>\mathrm{G}$ & x317 & 47.0 & H523D \\
\hline$C D C 7$ & NM_003503 & c. $1751 \mathrm{~A}>\mathrm{G}$ & x298 & 48.3 & N529S \\
\hline$C R E B B P$ & NM_001079846 & c. $2478 \mathrm{G}>\mathrm{T}$ & $\mathrm{x} 470$ & 54.7 & Q758H \\
\hline$E 2 F 1$ & NM_005225 & c. $1399 \mathrm{G}>\mathrm{A}$ & $\mathrm{x} 126$ & 30.2 & G420D \\
\hline$E I F 2 A K 4$ & NM_001013703 & c. $3268 \mathrm{G}>\mathrm{A}$ & x239 & 46.0 & $\mathrm{R} 1073 \mathrm{H}$ \\
\hline FERMT3 & NM_031471 & c. $2080 \mathrm{G}>\mathrm{T}$ & $\mathrm{x} 48$ & 56.3 & R644L \\
\hline$F O X O 3^{\mathrm{a}}$ & NM_201559 & c. $2013 \mathrm{G}>\mathrm{C}$ & x1001 & 41.3 & G566A \\
\hline$L R R K 2^{\mathrm{a}}$ & NM_198578 & c. $4314 \mathrm{G}>\mathrm{A}$ & x166 & 43.4 & $\mathrm{R} 1398 \mathrm{H}$ \\
\hline$M A P K 7^{\mathrm{a}}$ & NM_139033 & c. $2475 \mathrm{G}>\mathrm{A}$ & $\mathrm{x} 287$ & 61.7 & G697R \\
\hline$P D G F R B^{\mathrm{a}}$ & NM_002609 & c. $3441 \mathrm{G}>\mathrm{A}$ & x129 & 35.7 & R991H \\
\hline PTCH1 & NM_000264 & c. $4423 \mathrm{C}>\mathrm{T}$ & $x 60$ & 63.3 & P1412L \\
\hline$P T C H 2^{\mathrm{a}}$ & NM_003738 & c. $2975 \mathrm{C}>\mathrm{T}$ & $\times 502$ & 61.6 & T988M \\
\hline$R P S 6 K A 2^{\mathrm{a}}$ & NM_021135 & c. $1202 \mathrm{G}>\mathrm{A}$ & x293 & 41.0 & R328Q \\
\hline$R P S 6 K B 2^{\mathrm{a}}$ & NM_003952 & c. $882 \mathrm{C}>\mathrm{T}$ & $\mathrm{x} 446$ & 54.0 & P267L \\
\hline SOS2 & NM_006939 & c. $668 \mathrm{~A}>\mathrm{T}$ & x338 & 49.1 & E190D \\
\hline STK4 & NM_006282 & c. $962 \mathrm{G}>\mathrm{A}$ & x7195 & 49.1 & R291Q \\
\hline$S T K 10^{\mathrm{a}}$ & NM_005990 & c. $2201 \mathrm{G}>\mathrm{A}$ & $x 462$ & 58.7 & $\mathrm{R} 634 \mathrm{H}$ \\
\hline TRIM33 & NM_015906 & c. $1049 \mathrm{C}>\mathrm{T}$ & X599 & 44.9 & A322V \\
\hline
\end{tabular}

${ }^{\mathrm{a}}$ Registered in the 1000 genome project database.

A

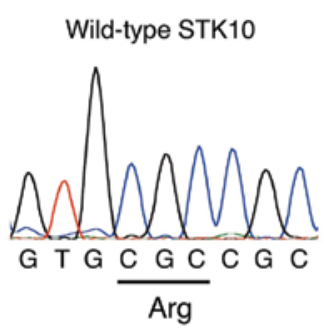

B

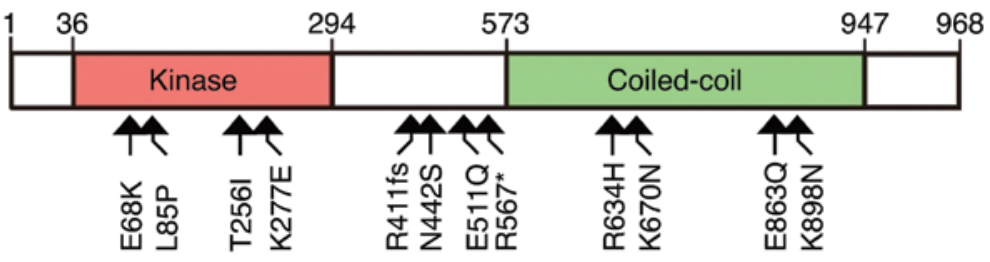

Figure 1. Identification of STK10(R634H) in a PTCL specimen. (A) Genomic DNA corresponding to the R634 position of STK10 protein was amplified by PCR from a PTCL specimen, and subjected to Sanger sequencing. Nucleotide and amino acid substitutions are shown red. (B) Schematic representation of STK10 protein structure with amino acid number shown at the top. The positions of amino acid changes reported in this study are depicted at the bottom.

Screening of missense mutations, insertions, deletions and fusion genes through our in-house computational pipeline (8) revealed a total of 19 nonsynonymous mutations but no indels or gene fusions, which were further confirmed by Sanger sequencing (Table I). It should be noted, however, that 9 of the 19 nonsynonymous mutations were recently registered as single nucleotide polymorphisms (SNPs) in the 1000 genome project (http://www.1000genomes.org) after our initial analysis.
One of the 19 nonsynonymous mutations was a nucleotide substitution of G-to-A at position 2201 of human STK10 cDNA (GenBank accession number, NM_005990) that was later deposited as an SNP (rs115974403). Sequencing of this position in genomic DNA of the same specimen further confirmed this substitution, which results in replacement of an arginine residue at amino acid position 634 with a histidine residue (R634H) (Fig. 1A). We also searched STK10 mutations among our human cancer specimens $(n=76)$, cancer cell lines 
A

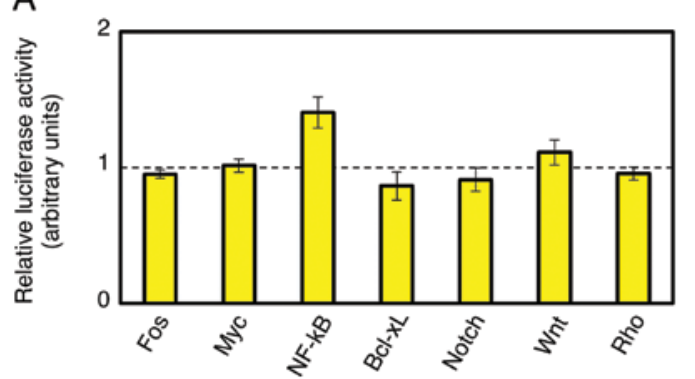

B

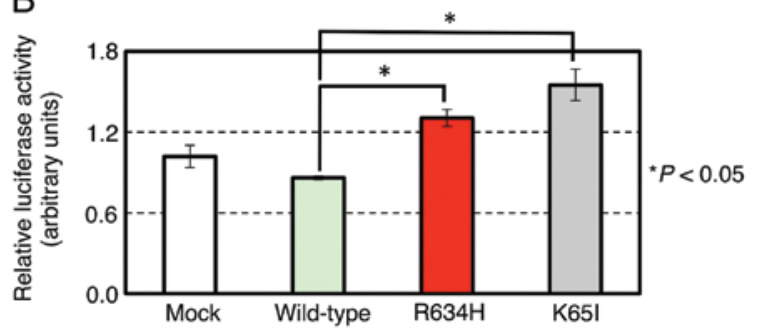

C

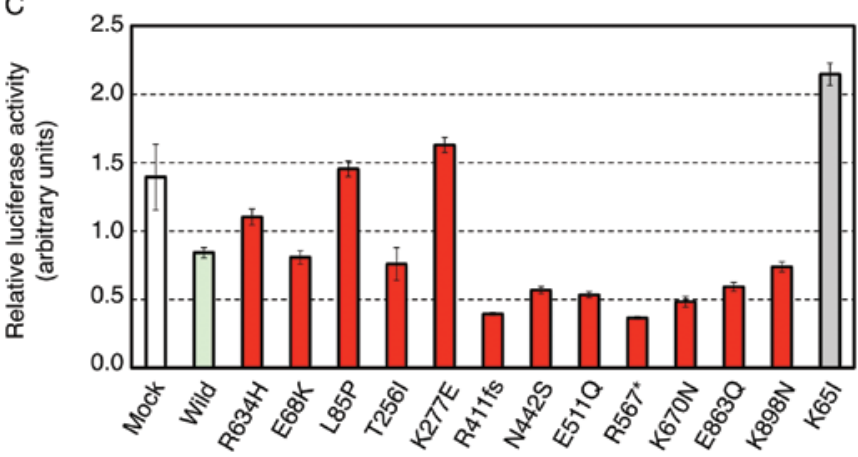

Figure 2. Activation of the NF- $\mathrm{BB}$ signaling by STK10 mutants. (A) HEK293 cells were transfected with an expression vector for the wild-type STK10 or STK10(R634H) together with pGL-4.70 and various reporter plasmids. Luciferase activities were determined at $48 \mathrm{~h}$ after transfection, and the firefly luciferase activities were normalized to those of the Renilla luciferase. The graph shows the STK10(R634H)-triggered luciferase activity for each pathway normalized by that with the wild-type. Data are the means $\pm \mathrm{SD}$ from three separate experiments. (B and C) As in (A), the NF- $\kappa \mathrm{B}$ reporter activities induced by various STK10 mutants were determined in HEK293 cells. Data are the means \pm SD from three separate experiments. (D) HEK293 cells were transfected with an expression vector for the wild-type STK10 or the indicated STK10 mutants. Expression levels of the genes indicated at the top of each panel were quantified by real-time RT-PCR, and the relative expression level of each gene to GAPDH was normalized to that of the mocktransfected cells. Data are the means \pm SD from three separate experiments. (E) HEK293 cells were transfected with the expression plasmid for STK10 or STK10(K65I) together with the NF- $\mathrm{KB}$ reporter plasmid, and subjected to luciferase assays at $48 \mathrm{~h}$ after transfection. Cells transfected with an empty vector (Mock) were similarly analyzed. The same set of cells were either stimulated with $1 \mathrm{ng} / \mathrm{ml}$ of TNF- $\alpha$ for $6 \mathrm{~h}$ before harvest (the middle panel), or co-transfected with expression plasmids for IKK $\alpha, \operatorname{IKK} \beta$ and IKK $\gamma$ (+ IKK complex). Data are the means \pm SD from three separate experiments.

$(\mathrm{n}=56)$ and the COSMIC database for cancer genome mutations (Release v58, http://cancer.sanger.ac.uk/cancergenome/ projects/cosmic/), revealing additional 11 nonsynonymous mutations; E68K in a CML cell line K562, L85P, T256I, R411frameshift (R411fs), K670N and K898N in various specimens of ovarian cancer, K277E in a testicular cancer specimen, N442S in a pancreatic cancer cell line Pa21C, E511Q in a
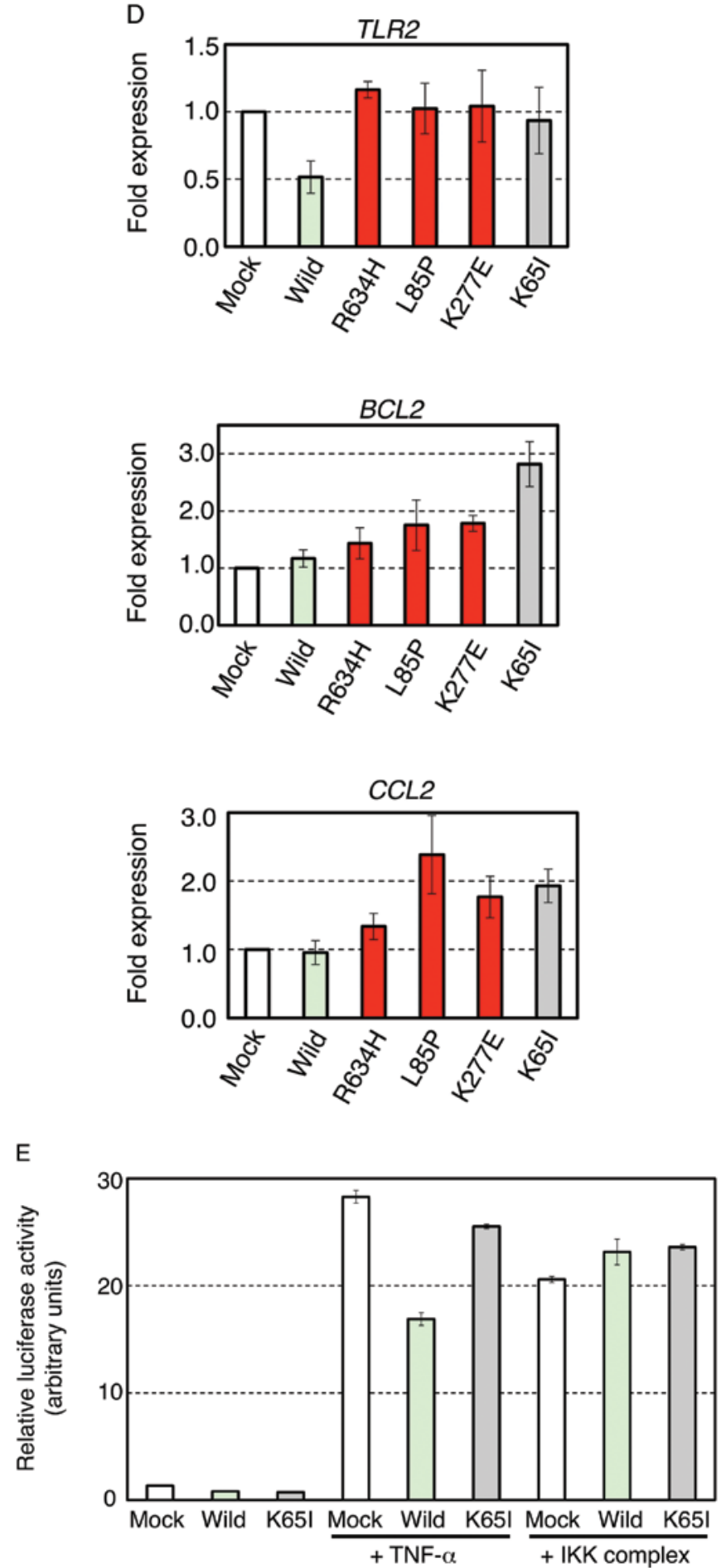

specimen of upper aerodigestive tract cancer, R567* in Jurkat, and E863Q in a lung cancer specimen (Fig. 1B).

Activation of $N F-\kappa B$ signaling by STK10 mutants. To examine whether STK10(R634H) affects intracellular signaling pathway related to Fos, Myc, NF- $\mathrm{B}$, Bcl-xL, Notch, Wnt or Rho, we examined the reporter activity for each pathway under the expression of the wild-type or the R634H mutant of STK10. As shown in Fig. 2A, the R634H mutant significantly elevated the $\mathrm{NF}-\kappa \mathrm{B}$ reporter activity compared to the wild-type $(\mathrm{P}<0.05$, Student's t-test), but did not affect the other signaling systems. To further investigate the role of STK10 


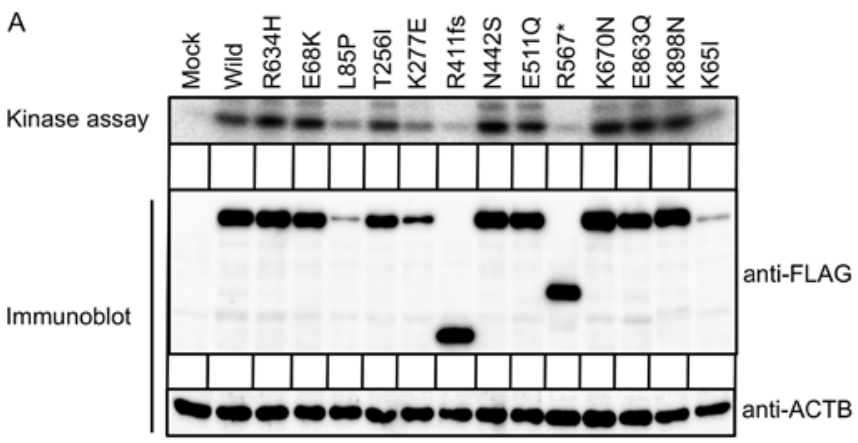

B

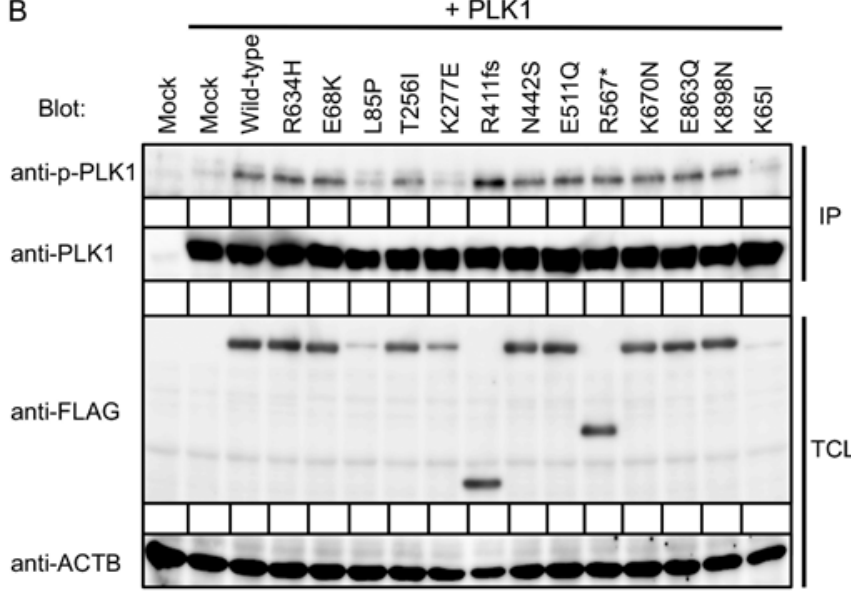

Figure 3. (A) Kinase activities of STK10 mutants. HEK293 cells were transfected with an expression vector for FLAG-tagged wild-type or mutant forms of STK10. Total cell lysates (TCL) were immunoprecipitated with anti-FLAG antibody, and subjected to an in vitro kinase assay with histone $\mathrm{H} 2 \mathrm{~A}$ as a substrate (top panel). TCL of each fraction was separately immunoblotted with antibodies to FLAG or ACTB (lower panel). Cells transfected with an empty vector were similarly analyzed (Mock). (B) HEK293 cells were transfected with an expression plasmid for FLAG-tagged wild-type or mutant forms of STK10 together with that for PLK1 as indicated at the top. TCL was subjected to immunoprecipitation (IP) with antibodies to PLK1, and immunoblotted with the same antibodies (anti-PLK1) or antibodies to the phosphorylated forms of PLK1 (anti-p-PLK1) (top panel). TCLs of the same samples were separately probed with antibodies to either FLAG or ACTB (lower panel).

in the NF- $\mathrm{KB}$ signaling, we generated cDNA for a kinasedead mutant (K65I) of STK10, and examined its effects on the NF- $\kappa B$ signaling. Of note, the wild-type STK10 rather suppressed the NF- $\mathrm{KB}$ pathway, but the R634H mutation abolished such effect, suggesting that STK10 is a negative regulator for intracellular NF- $\mathrm{KB}$ activity (Fig. 2B). This hypothesis was further reinforced by the fact that the kinase-inactive K65I mutant markedly elevates the NF- $\mathrm{BB}$ reporter signaling. We further asked the effects of other STK10 mutants on NF- $\mathrm{kB}$, and revealed that the $\mathrm{L} 85 \mathrm{P}$ and $\mathrm{K} 277 \mathrm{E}$ mutants found in the COSMIC database clearly induced NF-kB (Fig. 2C). Noteworthy, these mutations were somatically acquired in ovarian and testicular cancers, respectively. The other STK10 mutations did not affect NF- $\mathrm{\kappa B}$ signaling (Fig. $2 \mathrm{C}$ and data not shown).

To further confirm the effects of STK10 mutants on the $\mathrm{NF}-\mathrm{\kappa B}$ activation, expression levels of its target genes were quantified among cells expressing the wild-type or mutant forms of STK10 (Fig. 2D). Consistent with the results of luciferase assay, L85P, K277E, R634H and K65I mutants each induced the expression of NF- $\mathrm{KB}$ target genes including TLR2, BCL2 and $C C L 2$, compared to the wild-form. These results indicate that polymorphism as well as somatic mutations of STK10 may regulate $\mathrm{NF}-\mathrm{\kappa B}$ signaling in various human cancers.

It is not clear yet how STK10 regulates the NF- $\kappa B$ pathway. When HEK293 cells were stimulated with tumor necrosis factor (TNF)- $\alpha, \mathrm{K} 65 \mathrm{I}$ amino acid change cancelled the STK10-mediated NF-кB suppression (Fig. 2E). However, such regulation was not observed on the IKK complex-driven NF- $\kappa B$ activation, indicating that STK10 may interact with the NF- $\kappa \mathrm{B}$ pathway between TNF receptors and the IKK complex.

Kinase activities of STK10 mutants. To examine if the STK10 mutations directly affect its enzymatic activity, FLAGtagged wild-type or mutant forms of STK10 was expressed in HEK 293 cells, and subjected to immunoprecipitation with antibodies to FLAG, followed by an in vitro kinase assay with histone $\mathrm{H} 2 \mathrm{~A}$ as an exogenous substrate. As shown in Fig. 3A, while the wild-type STK10 clearly phosphorylates histone H2A, the L85P, K277E and K65I mutants remarkably attenuate the substrate phosphorylation. We could not, however, observe a notable difference between the wild-type and the R634H mutant in their ability of $\mathrm{H} 2 \mathrm{~A}$ phosphorylation, suggesting that R634H mutation may regulate the NF- $\mathrm{KB}$ signaling not through suppressing its enzymatic activity but through affecting its interaction to other proteins.

Of note, the R411fs and R567* mutants did not phosphorylate histone $\mathrm{H} 2 \mathrm{~A}$, indicating the coiled-coil domain and/or carboxyl terminal end of STK10 (Fig. 1B) is indispensable for histone phosphorylation. Immunoblot analysis with antibodies to FLAG revealed that protein amounts of the L85P, K277E and K65I mutants were markedly reduced, which may account for the decreased phosphorylation of histone $\mathrm{H} 2 \mathrm{~A}$.

Since STK10 is also known to phosphorylate PLK1 (14), we further investigated the phosphorylation level of PLK1 when co-expressed with the wild-type or mutant forms of SKT10 (Fig. 3B). Consistent with the result of the in vitro kinase assay, phosphorylation of PLK1 was reduced under the presence of the L85P, K277E or K65I mutant. Again, R634H mutation did not significantly affect the PLK1 phosphorylation. Strikingly, however, both of R411fs and R567* mutants could phosphorylate PLK1 equally to, or greater than, the wild-type. The carboxyl-terminal half of STK10 may, thus, be dispensable for the phosphorylation of PLK1 in vivo.

Anti-apoptotic function of STK10 mutants. NF- $\mathrm{B}$ plays pivotal roles in inflammatory and anti-apoptotic responses in cells, and constitutive NF- $\mathrm{KB}$ activation has been recognized as a hallmark of several lymphoid malignancies (15). Given the NF- $\mathrm{KB}$-activating potential of STK10 mutants, we then asked whether such mutants also regulate cell apoptosis. Glucocorticoids are known to be a potent apoptosis-inducer in lymphocytes, and have been used in therapeutic regimens for lymphoid malignancies (15). As shown in Fig. 4A, treatment of a mouse T-cell line, CTLL-2, with dexamethasone inhibited cell proliferation, and, importantly, introduction of the wild-type STK10 markedly augmented such effect, again confirming its tumor-suppressive role. In contrast, introduction 

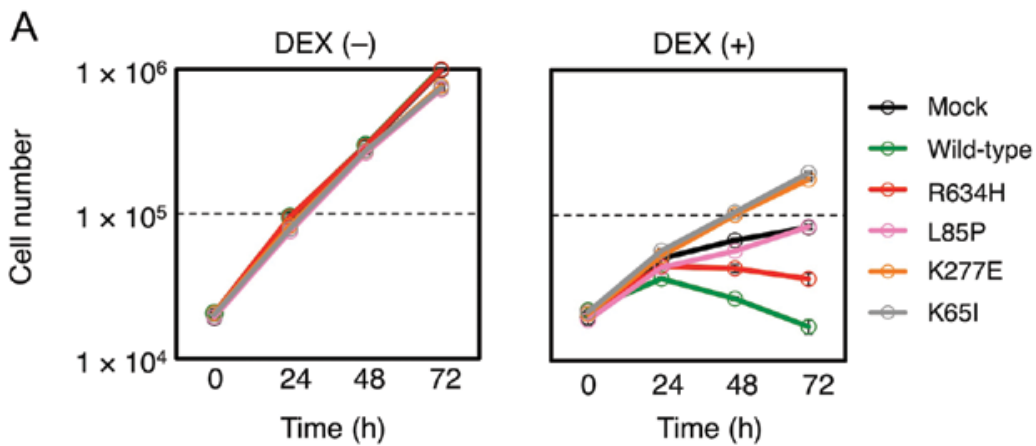

B

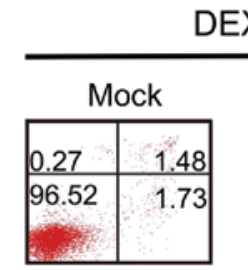

$\operatorname{DEX}(-)$

Wild-type

\section{$\mathrm{R} 634 \mathrm{H}$}

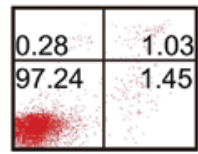

K277E

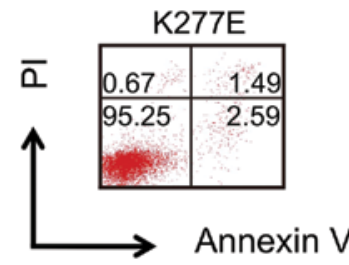

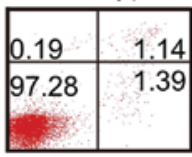

L85P

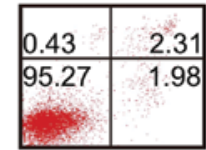

$\mathrm{K} 65 \mathrm{I}$

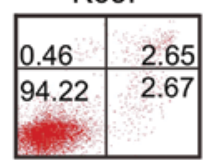

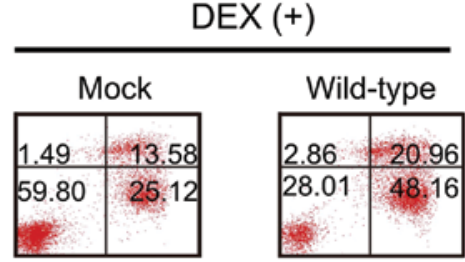

$\mathrm{R} 634 \mathrm{H}$
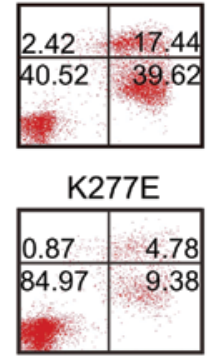

L85P

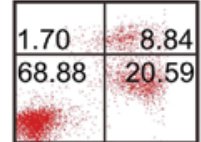

K65I

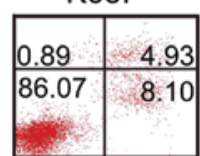

Figure 4. Anti-apoptotic effects of the STK10 mutants. (A) CTLL-2 cells expressing the wild-type STK10 or the indicated STK10 mutants were cultured with $2 \mathrm{ng} / \mathrm{ml}$ of IL-2 either in the absence (-) or presence (+) of $1 \mu \mathrm{M}$ dexamethasone (DEX). The number of viable cells was determined at the indicated times shown at the bottom. Data are means \pm SD from three separate experiments. CTLL-2 cells infected with mock retrovirus were similarly analyzed (Mock). (B) CTLL-2 cells at $72 \mathrm{~h}$ in (A) were collected and stained with Annexin V/PI, followed by flow cytometry. Positive or negative fractions for Annexin V or PI staining are shown as percentage for each experiment.

of NF- $\kappa \mathrm{B}$-activating mutants restored cell growth, in a parallel manner to their NF- $\mathrm{B}$-activating potential.

To determine whether the growth-inhibitory effect thus observed was related to apoptosis, we conducted the Annexin V/ PI staining assay (Fig. 4B). Although an increase in the Annexin V-positive fraction was apparent in dexamethasone-treated, wild-type STK10-expressing CTLL-2 cells, introduction of the L85P, K277E, R634H or K65 mutant of STK10 significantly suppressed apoptosis. Thus, a part of polymorphism/somatic mutations within STK10 not only activate the NF- $\mathrm{BB}$ pathway, but directly exert anti-apoptotic function.

Multiplex deep sequencing of STK10 in PTCL specimens. As it was demonstrated that some STK10 mutations induce $\mathrm{NF}-\kappa \mathrm{B}$ activation and render resistance to dexamethasone-induced apoptosis, we further searched for STK10 mutations in genomic DNAs of 92 PTCL specimens. It should be noted, however, that PTCL specimens frequently contain numerous normal cells in addition to neoplastic cells, which makes it difficult to determine the presence or absence of STK10 mutations with conventional Sanger sequencing. We therefore chose a deep sequencing approach with the GAIIx system (16), leading to the isolation of one specimen carrying STK10(R634H) (data not shown).

\section{Discussion}

STK10 is a member of the STE20 family of serine/threonine kinases that are involved in a variety of intracellular functions such as cell proliferation, regulation of apoptosis, rearrangement of the cytoskeleton and cell motility (17). While the Lok kinase, a mouse homolog of STK10, was shown to be highly expressed in lymphocytes (18), expression of STK10 may be detected in other tissues as well, including brain, colon, thymus, kidney, liver, small intestine and lung (14).

In this study, we identified STK10(R634H) in a PTCL specimen, and demonstrated that the R634H mutation induces $\mathrm{NF}-\kappa \mathrm{B}$ activation. We also demonstrated, for the first time, that the wild-type STK10 is a negative regulator for $\mathrm{NF}-\kappa \mathrm{B}$, and may be a tumor suppressor since it strongly augments dexamethasone-driven apoptosis in T-lymphocytes. Given the fact that STK10(R634H) attenuates cell apoptosis, such polymorphism may predispose lymphomagenesis. Indeed, through a mutation search of additional PTCL specimens $(n=92)$, we 
identified another PTCL case positive for the R634H polymorphism. While the cohort size is still small, frequency $(2 / 92=2.2 \%)$ of this polymorphism in PTCL is significantly over-represented compared to that $(1 / 2385=0.04 \%)$ in the 1000 genome database $\left(\mathrm{P}=4.0 \times 10^{-3}\right.$, Fisher's exact test $)$.

Noteworthy, some of the somatic mutations within STK10 found in epithelial tumors have more profound effects on the $\mathrm{NF}-\kappa \mathrm{B}$ regulation and cell apoptosis than $\mathrm{R} 634 \mathrm{H}$. Thus, dysfunction of STK10 through somatic mutations is likely a novel, driver event in human carcinogenesis. Restoration of STK10 function or/and suppression of $N F-\kappa B$ in the tumors positive for STK10 mutations can be a novel strategy for raising effective molecular targeted therapies against human cancer.

\section{Acknowledgements}

This study was supported in part by grants for Third-Term Comprehensive Control Research for Cancer and for Research on Human Genome Tailor-made from the Ministry of Health, Labor and Welfare of Japan, by Grants-in-Aid from the Japan Society for the Promotion of Science, from The Yasuda Medical Foundation, from The Sagawa Foundation for Promotion of Cancer Research, and from The Mitsubishi Foundation.

\section{References}

1. Lynch TJ, Bell DW, Sordella R, et al: Activating mutations in the epidermal growth factor receptor underlying responsiveness of non-small-cell lung cancer to gefitinib. N Engl J Med 350: 2129-2139, 2004.

2. Soda M, Choi YL, Enomoto M, et al: Identification of the transforming $E M L 4-A L K$ fusion gene in non-small-cell lung cancer. Nature 448: 561-566, 2007.

3. Mok TS, Wu YL, Thongprasert S, et al: Gefitinib or carboplatinpaclitaxel in pulmonary adenocarcinoma. N Engl J Med 361: 947-957, 2009.

4. Kwak EL, Bang YJ, Camidge DR, et al: Anaplastic lymphoma kinase inhibition in non-small-cell lung cancer. $\mathrm{N}$ Engl J Med 363: 1693-1703, 2010.
5. Foss FM, Zinzani PL, Vose JM, Gascoyne RD, Rosen ST and Tobinai K: Peripheral T-cell lymphoma. Blood 117: 6756-6767, 2011.

6. Vose J, Armitage $\mathrm{J}$ and Weisenburger D: International peripheral T-cell and natural killer/T-cell lymphoma study: pathology findings and clinical outcomes. J Clin Oncol 26: 4124-4130, 2008.

7. Gambacorti-Passerini C, Messa C and Pogliani EM: Crizotinib in anaplastic large-cell lymphoma. N Engl J Med 364: 775-776, 2011.

8. Ueno T, Yamashita Y, Soda M, et al: High-throughput resequencing of target-captured cDNA in cancer cells. Cancer Sci 103: 131-135, 2012

9. Hu Q, Milfay D and Williams LT: Binding of NCK to SOS and activation of ras-dependent gene expression. Mol Cell Biol 15: 1169-1174, 1995.

10. Takeshita T, Arita T, Higuchi M, et al: STAM, signal transducing adaptor molecule, is associated with Janus kinase and involved in signaling for cell growth and c-myc induction. Immunity 6 : 449-457, 1997.

11. Grillot DAM, Gonzalez-Garcia M, Ekhterae D, et al: Genomic organization, promoter region analysis, and chromosome localization of the mouse $b c l-x$ gene. J Immunol 158: 4750-4757, 1997.

12. Kurooka H, Kuroda $\mathrm{K}$ and Honjo T: Roles of the ankyrin repeats and C-terminal region of the mouse notch1 intracellular region. Nucleic Acids Res 26: 5448-5455, 1998.

13. Hill CS, Wynne J and Treisman R: The Rho family GTPases RhoA, Rac1, and CDC42Hs regulate transcriptional activation by SRF. Cell 81: 1159-1170, 1995.

14. Walter SA, Cutler RE Jr, Martinez R, Gishizky M and Hill RJ: Stk10, a new member of the polo-like kinase kinase family highly expressed in hematopoietic tissue. J Biol Chem 278: 18221-18228, 2003.

15. Jost PJ and Ruland J: Aberrant NF-kappaB signaling in lymphoma: mechanisms, consequences, and therapeutic implications. Blood 109: 2700-2707, 2007.

16. Choi YL, Soda M, Ueno T, et al: Oncogenic MAP2K1 mutations in human epithelial tumors. Carcinogenesis 33: 956-961, 2012.

17. Dan I, Watanabe NM and Kusumi A: The Ste20 group kinases as regulators of MAP kinase cascades. Trends Cell Biol 11: 220-230, 2001

18. Kuramochi S, Moriguchi T, Kuida K, Endo J, Semba K, Nishida E and Karasuyama H: LOK is a novel mouse STE20-like protein kinase that is expressed predominantly in lymphocytes. J Biol Chem 272: 22679-22684, 1997. 